Decreased homotopic interhemispheric functional connectivity in children with

\title{
autism spectrum disorder
}

3 Running title: Homotopic connectivity in autistic children

4 Shuxia Yao*, Menghan Zhou, Yuan Zhang, Feng Zhou, Qianqian Zhang, Zhongbo Zhao,

5 Xi Jiang, Xiaolei Xu, Benjamin Becker, Keith M. Kendrick*

6 The Clinical Hospital of Chengdu Brain Science Institute, MOE Key Laboratory for

7 NeuroInformation, Center for Information in Medicine, University of Electronic Science

8 and Technology of China, Chengdu, Sichuan 611731, China

$9 *$ Corresponding authors

10 Correspondence: Shuxia Yao or Keith M. Kendrick, No.2006, Xiyuan Ave., West Hi-

11 Tech Zone, Chengdu, Sichuan 611731, China. Fax: +86-28-61830811. Email:

12 yaoshuxia@uestc.edu.cn or k.kendrick.uestc@gmail.com

\section{Acknowledgments}

14 This study was supported by the National Natural Science Foundation of China (NSFC)

15 grants (grant number: 31700998, 31530032) and by the Department of Science and

16 Technology of Guangdong Province (grant number: 2018B030335001). The primary

17 funding source for ABIDE-II is from NIMH 5R21MH107045.

18 Declaration of interest: The authors declare no conflict of interest.

19 Lay Summary: Homotopic interhemispheric functional connectivity plays an important

20 role in synchronizing activity between the two hemispheres and is altered in adults and 
21 adolescents with autism spectrum disorder (ASD). In the present study focused on

22 children with ASD, we have observed similar decreased homotopic connectivity in

23 extensive brain networks, suggesting that alterations in homotopic interhemispheric

24 connectivity may occur early in ASD and be a useful general biomarker across ages. 


\section{Abstract}

27 While a number of functional and structural changes occur in large-scale brain

28 networks in autism spectrum disorder (ASD), reduced interhemispheric resting state

29 functional connectivity (rsFC) between homotopic regions may be of particular

30 importance as a biomarker. ASD is an early-onset developmental disorder and neural

31 alterations are often age-dependent, reflecting dysregulated developmental trajectories,

32 although no studies have investigated whether homotopic interhemispheric rsFC

33 alterations occur in ASD children. The present study conducted a voxel-based homotopic

34 interhemispheric rsFC analysis in 146 SD and 175 typically developing children under

35 age 10 and examined associations with symptom severity in the Autism Brain Imaging

36 Data Exchange datasets. Given the role of corpus callosum (CC) in interhemispheric

37 connectivity and reported CC volume changes in ASD we additionally examined whether

38 there were parallel volumetric changes in ASD children. Results demonstrated decreased

39 homotopic rsFC in ASD children in the medial prefrontal cortex, precuneus and posterior

40 cingulate cortex of the default mode network (DMN), the dorsal anterior cingulate cortex

41 of the salience network, the precentral gyrus and inferior parietal lobule of the mirror

42 neuron system, the lingual, fusiform and inferior occipital gyri of the visual processing

43 network and thalamus. Symptom severity was associated with homotopic rsFC in regions

44 in the DMN and visual processing network. There were no significant $\mathrm{CC}$ volume

45 changes in ASD children. The present study shows that reduced homotopic 
46 interhemispheric rsFC in brain networks in ASD adults/adolescents is already present in

47 children of 5-10 years old and further supports their potential use as a general ASD

48 biomarker.

49 Keywords: Autism Spectrum Disorder; Children; Resting State; Homotopic

50 Interhemispheric Functional Connectivity; Corpus Callosum 


\section{Introduction}

53 Autism spectrum disorder (ASD) is a heterogeneous neurodevelopmental disorder with

54 core symptomatology generally characterized by deficits in social interaction, verbal and

55 nonverbal communication and restrictive or stereotyped behavior (Kanner, 1943).

56 Behavioral studies have revealed a variety of impairments in social-cognitive and

57 affective domains in ASD patients, including deficits in joint attention (Charman, 2003),

58 weak central coherence (Happé \& Frith, 2006), avoidance of eye contact (Madipakkam,

59 Rothkirch, Dziobek, \& Sterzer, 2017), difficulties in face recognition (Fedor et al., 2018)

60 and diminished interest and motivation in social interactions (Chevallier, Grèzes,

61 Molesworth, Berthoz, \& Happé, 2012; Morrison et al., 2017). Task-based neuroimaging

62 studies have demonstrated that these deficits are mediated by dysfunctions in large-scale

63 brain systems sub-serving social-cognitive and affective processes including the default

64 mode network (DMN), mirror neuron system (MNS), theory of mind (ToM), and

65 executive control networks (Dapretto et al., 2006; Herringshaw, Kumar, Rody, \& Kana,

66 2018; Oberwelland et al., 2017; von dem Hagen, Stoyanova, Rowe, Baron-Cohen, \&

67 Calder, 2014; Wang, Dapretto, Hariri, Sigman, \& Bookheimer, 2004).

68 The behavioral deficits in ASD and the need for diagnostics at an early age limit the

69 application of task-based fMRI assessments in ASD. Consequently, resting state fMRI

70 (rs-fMRI), which has the advantage of being task-free and providing comparable

71 measures across different studies and sites, has been considered as a promising approach 
72 for investigating neural abnormalities and for providing possible promising biomarkers in

73 ASD. In line with the task-based fMRI findings, ASD-associated alterations in the

74 intrinsic organization of the brain as assessed by resting state functional connectivity

75 (rsFC) have been found in the DMN, MNS, ToM and executive control networks (Chen,

76 Yang, Wu, Chuang, \& Huang, 2019; Fishman, Keown, Lincoln, Pineda, \& Müller, 2014;

77 Funakoshi et al., 2016; Monk et al., 2009), suggesting that large-scale aberrant functional

78 organization occurs both in a task-free and task-based conditions in ASD. Additional

79 brain networks showing rsFC abnormities in autistic patients have been reported in

80 salience, mesolimbic reward and language networks (Lee, Park, James, Kim, \& Park,

81 2017; Supekar et al., 2018; Uddin et al., 2013). Furthermore, atypical developmental

82 trajectories in the functional and structural organization of these networks have also been

83 found in ASD (Ecker, Bookheimer, \& Murphy, 2015; Geschwind \& Levitt, 2007;

84 Washington et al., 2014).

85 Based on altered functional connectivity patterns, ASD has been proposed as a

86 developmental dysfunctional connectivity syndrome with local overconnectivity but

87 long-distance underconnectivity (Courchesne \& Pierce, 2005; Geschwind \& Levitt, 2007;

88 Lau, Leung, \& Lau, 2019). Recent studies have focused on interhemispheric functional

89 connections particularly the homotopic ones, which may be of great importance in ASD.

90 Homotopic interhemispheric functional connectivity is a measurement of the rsFC

91 between each voxel in one hemisphere and its mirrored counterpart in the opposite 
92 hemisphere (Stark et al., 2008; Zuo et al., 2010). As a key characteristic of the intrinsic

93 functional architecture of the brain, homotopic interhemispheric functional connections in

94 the brain are exceptionally strong and may therefore play an important role in

95 synchronizing activity between the two hemispheres (Stark et al., 2008). Anderson et al.

96 (2011) first investigated the homotopic interhemispheric rsFC in ASD and found

97 decreased homotopic interhemispheric rsFC between sensorimotor cortex, insula,

98 fusiform gyrus, superior temporal gyrus, and superior parietal lobule in ASD compared to

99 typically developing (TD) controls. Two other studies using the large Autism Brain

100 Imaging Data Exchange (ABIDE) multisite datasets (ABIDE-I or -II), which have the

101 advantage of greater statistical power, confirmed homotopic interhemispheric rsFC

102 alterations in ASD, particularly in the interhemispheric communication of the posterior

103 cingulate cortex (PCC), insula and thalamus (Di Martino et al., 2014 - ABIDE-I) and

104 across a variety of large-scale networks (King et al., 2019; ABIDE-I and -II). Including

105 gender as an additional covariate, Li et al. (2019) using the ABIDE-I dataset further

106 confirmed decreased homotopic interhemispheric connectivity in ASD subjects and

107 reported associations between interhemispheric functional connectivity in the PCC, insula

108 and superior temporal gyrus and social communication impairment severity using the

109 Autism Diagnostic Observation Schedule (ADOS). Thus, interhemispheric functional

110 connectivity changes may represent a particular promising biomarker for discriminating

111 ASD from TD individuals (Li et al., 2019). Note that some ASD-related rsFC changes are 
112 age-dependent (Hull, Jacokes, Torgerson, Irimia, \& van Horn, 2017) and there is also

113 evidence that homotopic interhemispheric connectivity shows different developmental

114 trajectories in ASD compared with TD individuals (Kozhemiako et al., 2018). It is

115 therefore possible that interhemispheric communication dysfunctions may also represent

116 a result of dysregulated developmental trajectories. Although interhemispheric

117 connectivity alterations have been investigated in ASD patients, subjects used in previous

118 studies, including the ones using ABIDE datasets, are primarily high functioning

119 adults/adolescents with ASD (e.g., mean = 22.4 years old in Anderson et al., 2011 and

12017.39 years old for ABIDE-I). However, despite the neurodevelopmental nature of the

121 disorder (Hull et al., 2017; Kozhemiako et al., 2018), no study to date has specifically

122 examined homotopic interhemispheric rsFC changes in ASD children.

123 To shed new light on the utility of using homotopic interhemispheric rsFC

124 alterations as biomarkers for ASD in general and as early biomarkers to determine ASD

125 in childhood in particular, the present study has therefore conducted a voxel-based

126 functional connectivity analysis to investigate whether alterations in homotopic

127 interhemispheric rsFC are already present in ASD children and whether the changes are

128 associated with ASD symptom severity. Furthermore, as the primary structure connecting

129 the left and right brain hemispheres, corpus callosum (CC) volumes have been considered

130 as an index of interhemispheric connectivity (Vidal et al., 2006; Wegiel et al., 2018) and

131 volumetric CC alterations have also been associated with ASD (Anderson et al., 2011; 
132 Frazier \& Hardan, 2009). The present study thus additionally examined whether there

133 were parallel CC volume changes in ASD children and, if so, associations with symptom

134 severity.

\section{Methods}

\section{Participants}

137 Resting-state fMRI and T1-weighted anatomical MRI images of 476 children under the

138 age of 10 years $($ mean $=8.57, \mathrm{SD}=0.947$; range: $5.3-10)$ were obtained from the

139 ABIDE-I and -II datasets (http://fcon_1000.projects.nitrc.org/indi/abide/ for more

140 information). The threshold of 10 years old was selected based on definition of age range

141 for life phases from the American Academy of Pediatrics (Hagan, Shaw, \& Duncan,

142 2008) and the World Health Organization (https://www.who.int/southeastasia/health-

143 topics/adolescent-health). Consistent with Li et al. (2019), subjects were excluded in

144 cases where preprocessing was not successful and head motion was excessive. Subjects

145 were excluded in cases where: (a) fMRI images could not be successfully preprocessed,

146 as determined by visual inspection of the normalization success; (b) mean framewise

147 displacement (FD) was $>0.5 \mathrm{~mm}$; (c) the percentage of FD >0.5 mm was over $25 \%$.

148 After excluding subjects based on these criteria, data from sites with less than 4 subjects

149 remaining were further deleted from the subsequent analyses. To further control for head

150 motion, we matched head motion (mean FD) in the 2 groups (ASD group: mean $=0.20$,

$151 \mathrm{SD}=0.096$; control group: mean $=0.18, \mathrm{SD}=0.097 ; \mathrm{t}(319)=1.48, \mathrm{p}=0.139)$. These 
152 steps led to 146 subjects (50 from ABIDE-I) in the ASD group and 175 subjects (62 from

153 ABIDE-I) in the TD control group being included in the final interhemispheric functional

154 connectivity analysis, with the majority of subjects in the two groups (96 ASD vs. 113

155 control) being from the ABIDE-II dataset. Subject demographic information is reported

156 in Supplementary Table S1 and clinical information of subjects with available scores on

157 symptom severity as measured by ADOS (module 3) were reported in Supplementary

158 Table S2 $(\mathrm{N}=70)$. To increase transparency and replicability, a full list of subject IDs

159 was also reported in Supplementary Table S3 and Table S4 for ABIDE-I and -II

160 respectively. The data from the ABIDE datasets was fully anonymized. All contributions

161 in this project were based on studies approved by local Institutional Review Boards.

162 Written informed consent was obtained from all subjects or carers.

\section{Data Analyses}

\section{Interhemispheric rsFC Analyses}

165 Interhemispheric rsFC analyses were similar to Li et al. (2019). Resting-state fMRI

166 images were preprocessed using the Data Processing Assistant for Resting-State fMRI

167 (DPARSF 2.3) software (Yan \& Zang, 2010; http://www.restfmri.net) for each site

168 separately following a standardized procedure (Yan et al., 2013). After discarding the

169 first 10 images, pre-processing included slice timing correction, realignment,

170 segmentation and spatial normalization. Segmentation was conducted based on a

171 pediatric tissue probabilistic map created by the Template-O-Matic Toolbox while taking 
172 age and sex of children used in the present study into consideration (Wilke, Holland,

173 Altaye, \& Gaser, 2008). The images were then normalized to the Montreal Neurological

174 Institute (MNI) template. Head-motion parameters (using the Friston 24-parameter

175 model; Friston, Williams, Howard, Frackowiak, \& Turner, 1996; Satterthwaite et al.,

176 2013), global signal, white matter and cerebrospinal fluid were regressed out to control

177 for potential confounding artifacts. To further exclude residual effects of head motion, we

178 included the mean FD as a nuisance covariate in the group analysis (Power et al., 2014)

179 and matched the mean FD between the 2 groups as described above. Following

180 resampling with a $3 \mathrm{~mm} \times 3 \mathrm{~mm} \times 3 \mathrm{~mm}$ resolution, the pre-processed images were

181 spatially smoothed with a Gaussian kernel (8-mm full-width at half-maximum) and

182 filtered using a band pass filter $(0.01-0.08 \mathrm{~Hz})$. The homotopic interhemispheric

183 functional connectivity map was then generated based on a voxel-mirrored homotopic

184 connectivity (VMHC) analysis (Zuo et al., 2010) implemented in the DPARSF toolbox

185 (Yan \& Zang, 2010). As a voxel-based functional connectivity analysis approach, the

186 VMHC reflects rsFC between each voxel in one hemisphere and its mirrored counterpart

187 in the opposite hemisphere (Stark et al., 2008; Zuo et al., 2010). The homotopic

188 interhemispheric functional connectivity maps were then converted into Z-maps using

189 Fisher's z transformation for subsequent group level analyses. To promote a strict control

190 of center variability in the rsFC data the combatting batch effect (ComBat) harmonization

191 method was applied, an approach that has been shown to remove unwanted variation 
192 associated with site/scanner while preserving inter-site biological variability

193 (https://github.com/canlab/ComBatHarmonization\#id-section2). ComBat models site-

194 specific scaling factors and uses empirical Bayes to improve the estimation of site

195 parameters for small sample sizes. Previous studies have demonstrated that the ComBat

196 harmonization method is more advanced in controlling for site effects relative to

197 conventional regression methods for different imaging modalities and that it performs

198 well for multi-site imaging studies with only a few participants in each site or for

199 unbalanced sample size between studies or sites (Fortin et al., 2017, 2018; Johnson, Li, \&

200 Rabinovic, 2007; Yu et al., 2018).

$201 \quad$ For the group level analyses, a two-sample t-test was used to test group functional

202 connectivity differences between the ASD and TD children. To control for potential

203 confounding effects, gender, age, total brain volume (grey matter + white matter) and

204 head motion (mean FD) were included as nuisance covariates in the group analysis. A

205 threshold of $\mathrm{p}<0.05$ false discovery rate (FDR) corrected for multiple comparisons at the

206 voxel level was used in the whole-brain analysis and only clusters larger than 10 voxels

207 were reported. To increase the sensitivity to determine whether the changes in homotopic

208 interhemispheric rsFC observed in a sample predominatly including adolescents and

209 adults (Li et al., 2019) already occur in ASD children, we further applied a mask

210 encompassing regions that exhibited altered homotopic interhemispheric rsFC in these

211 samples from Li et al. (2019). To this end a mask was created based on the thresholded 
212 statistic map ( $\mathrm{p}_{\mathrm{FDR}}<0.05$; see Figure S1C) of group differences between ASD and TD

213 control groups as reported in Li et al. (2019).

214 We also conducted correlation analyses between the homotopic interhemispheric

215 connectivity strength and symptom severity using Pearson correlation coefficients. The

216 homotopic interhemispheric connectivity strength was extracted from a 6-mm sphere

217 centered on the peak voxel for regions showing altered homotopic interhemispheric

218 connectivity in the ASD relative to the control group. ADOS consisted of social,

219 communication, and stereotyped behavior subscales and total ADOS scores and scores

220 for each subscale were available for 70 ASD children in the ABIDE datasets.

221 Corpus Callosum Volume Analyses

222 Volume analyses of the CC were conducted on T1 data using the VBM8 toolbox

223 (http://dbm.neuro.unijena.de/vbm8/; Ashburner \& Friston, 2000) and kept consistent with

224 Li et al. (2019). Volume analyses of the CC were conducted on T1 data using the VBM8

225 toolbox (http://dbm.neuro.unijena.de/vbm8/; Ashburner and Friston, 2000) and kept

226 consistent with Li et al. (2019). T1 images were normalized to the Montreal Neurological

227 Institute (MNI) template and segmented into GM, WM and cerebrospinal fluid. Data

228 quality check was performed via the modules of "display one slice for all images" and

229 "check sample homogeneity using covariance". 10 subjects were further excluded due to

230 their overall covariance homogeneity being below 2 standard deviations from the mean.

231 The remaining 139 ASD subjects and 172 controls were entered into the final analysis. 
232 Segmented WM images from these subjects were then smoothed with a Gaussian kernel

233 of 8-mm full-width at half-maximum. Site effects on $\mathrm{CC}$ volumes were also controlled by

234 employing the ComBat harmonization method (Fortin et al., 2017, 2018; Johnson, Li, \&

235 Rabinovic, 2007; Yu et al., 2018).

236 Group differences in CC volume were tested using a two-sample t-test on smoothed

237 WM images. Gender, age and the total brain volume (TBV) were included as nuisance

238 covariates to control for potential confounding effects. The structural CC mask was

239 derived from the Talairach Daemon database atlas (Lancaster, 1997). Within the CC ROI,

240 a threshold of $\mathrm{p}<0.05$ FDR corrected at peak-level was set for multiple comparisons and

241 only clusters larger than 10 voxels reported.

\section{Results}

\section{Interhemispheric Functional Connectivity}

244 Two-sample t-tests were used to examine homotopic interhemispheric functional

245 connectivity differences between the ASD and TD children. Analyses on the whole brain

246 level revealed that ASD compared to TD children exhibited decreased homotopic

247 interhemispheric functional connectivity in two clusters located in the PCC $(\mathrm{MNI}=6$, -

$24845,12, \mathrm{t}=4.88, \mathrm{p}_{\mathrm{FDR}}<0.05$, voxels $=17 ; \mathrm{MNI}=6,-42,24, \mathrm{t}=4.77, \mathrm{p}_{\mathrm{FDR}}<0.05$, voxels

$249=25)$. Applying the mask generated from homotopic interhemispheric FC alterations

250 previously observed in ASD adolescents and adults (Li et al., 2019) additionally revelaed

251 decreased homotopic interhemispheric functional connectivity in regions of the DMN, 
252 including the PCC, the dorsal medial prefrontal cortex (mPFC), ventral mPFC, precuneus

253 and the angular gyrus; the anterior cingulate cortex (ACC) of the salience network; the

254 precentral gyrus and inferior parietal lobule (IPL) of the mirror neuron system; the visual

255 processing network, including the lingual, fusiform, inferior and middle occipital gyri,

256 inferior and middle temporal gyri; and thalamus (extending from PCC) $\left(\mathrm{p}_{\mathrm{FDR}}<0.05\right.$;

257 Figure 1 and Table 1) in ASD children. Categorization of regions into large-scale brain

258 networks was based on previous reviews and studies on the default mode network

259 (Raichle, 2015; Raichle et al., 2001; Seghier, 2013), the salience network (Menon \&

260 Uddin, 2010), the mirror neuron system (Iacoboni \& Dapretto, 2006; Rizzolatti \&

261 Craighero, 2004), and the visual processing network (Bogousslavsky, Miklossy, Deruaz,

262 Assal, \& Regli, 2017; Eickenberg, Gramfort, Varoquaux, \& Thirion, 2017; Machielsen,

263 Rombouts, Barkhof, Scheltens, \& Witter, 2000). Group difference on altered homotopic

264 interhemispheric functional connectivity between ASD and TD control groups for each

265 site was presented in supplemental Figure S2 using the PCC as an example. There were

266 no regions showing significantly stronger interhemispheric functional connectivity in the

267 ASD relative to TD groups using the same threshold ( $\left.\mathrm{p}_{\mathrm{FDR}}<0.05\right)$. 
272 small part ( 12.96\%) of the subject sample used in our previous study (Li et al., 2019),

273 we aimed at further controlling the possibility that subject overlap may have contributed

274 to the consistent findings between the two studies. To this end, we further conducted an

275 identical analysis using only ASD and TD children from the ABIDE-II dataset and found

276 a highly similar but less robust pattern (see Figure S1A and B), which may be due to the

277 smaller sample size when focusing only on the ABIDE-II dataset. Additionally, we also

278 confirmed that removing data from children in the ABIDE-I dataset did not influence the

279 pattern of results we had previously reported using data predominantly from adolescents

280 and adults (Li et al., 2019) (see Figure S1C and D).

\section{Correlations between Interhemispheric Functional Connectivity and Symptom}

282 Severity

283 Correlation analyses between homotopic interhemispheric connectivity strength and

284 symptom severity were first conducted for the total ADOS scores. Results revealed that

285 the total ASD symptom load (total ADOS scores) showed a significant positive

286 correlation with the homotopic interhemispheric functional connectivity strength of the

287 PCC $(\mathrm{MNI}=6,-45,12 ;$ Pearson $\mathrm{r}=0.268, \mathrm{p}=0.025 ;$ Figure 2A $)$, but a negative

288 correlation with the middle temporal gyrus (MTG; Pearson $r=-0.240, p=0.046$; Figure

289 2B). Further explorations using sub-scale scores revealed significant positive correlations

290 between the homotopic connectivity strengths of the PCC (MNI =6, -45, 12) and social

291 sub-scale scores (Pearson $r=0.254, p=0.034$; Figure 2C), and of the precuneus (Pearson 
$292 \mathrm{r}=0.237, \mathrm{p}=0.048$; Figure 2D) for the communication sub-scale scores. Homotopic

293 functional connectivity strengths of the PCC $(\mathrm{MNI}=6,-42,24$; Pearson $\mathrm{r}=0.252, \mathrm{p}=$

294 0.035; Figure 2E), the inferior temporal gyrus (ITG; Pearson $r=0.274, p=0.022$; Figure

$2952 \mathrm{~F})$, the precuneus (Pearson $\mathrm{r}=0.362, \mathrm{p}=0.002$; Figure $2 \mathrm{G}$ ) and the IPL (Pearson $\mathrm{r}=$

$2960.242, \mathrm{p}=0.043$; Figure $2 \mathrm{H}$ ) were also positively associated with stereotyped behavior

297 sub-scale scores. In contrast, significant negative correlations were found between

298 homotopic connectivity strengths of the MTG (Pearson $r=-0.250, p=0.037$; Figure 2I)

299 and the inferior occipital gyrus (IOG; Pearson $r=-0.237, p=0.048$; Figure $2 \mathrm{~J}$ ) and social

300 sub-scale scores. However, none of these correlations can survive family-wise error

301 correction for multiple comparisons $(\mathrm{p}<0.05 / 22=0.002$, where 22 is the total number of

302 regions showing significant altered homotopic connectivity in ASD as presented in Table 303 1).

*****Insert Figure 2 here $* * * *$

\section{Corpus Callosum Volume}

306 The ROI-based analysis of CC volume did not reveal any significant volume changes in

307 the ASD compared to the TD group at a threshold of $\mathrm{p}_{\mathrm{FDR}}<0.05$.

\section{Discussion}

309 The present study investigated homotopic interhemispheric rsFC alterations in ASD

310 children under 10 years of age and their association with symptom severity. Given that

311 the $\mathrm{CC}$ is the key fiber tract connecting the two hemispheres, changes in $\mathrm{CC}$ volumes 
312 were also examined. Results showed decreased homotopic interhemispheric functional

313 connectivity in regions of the DMN, the salience network, the mirror neuron system, the

314 visual processing network and thalamus in the ASD relative to the TD group, with

315 interhemispheric functional connectivity of regions mainly in the DMN and the visual

316 processing network being correlated with symptom severity. No significant CC volume

317 changes were found in ASD children relative to TD controls. Altered homotopic

318 functional connectivity may therefore be a potential biomarker for ASD in both children

319 and adults.

320 Previous studies investigating homotopic interhemispheric rsFC changes in ASD

321 mainly used subjects ranging from children to adults (Anderson et al., 2011; Di Martino

322 et al., 2014; King et al., 2019; Li et al., 2019). For example, subjects from the ABIDE-I

323 dataset used in most of these studies have an average age of 17.39 years with only

$32411.92 \%$ under the age of 10 . However, only focusing on ASD children aged under 10, the

325 present study using combined ABIDE-I and -II datasets found similar widespread

326 decreased homotopic interhemispheric rsFC patterns as reported in these previous

327 predominantly adult/adolescent-based studies. More specifically, the present study

328 replicated the majority of brain networks exhibiting decreased homotopic

329 interhemispheric connectivity in ASD patients reported by Li et al. (2019). These

330 included the PCC, the dorsal and ventral mPFC, precuneus and the angular gyrus of the

331 DMN, the dACC of the salience network, the precentral gyrus and IPL of the mirror 
332 neuron system, the lingual, fusiform and inferior occipital gyri of the visual processing

333 network and thalamus. These networks are associated with a variety of cognitive and

334 social/affective domains including self-referential/consciousness processing and theory of

335 mind (the DMN; Davey, Pujol, \& Harrison, 2016; Raichle, 2015; Saxe, Moran, Scholz, \&

336 Gabrieli, 2006), cognitive control and emotion regulation (dACC; MacDonald, Cohen,

337 Stenger, \& Carter, 2000; Stevens, Hurley, \& Taber, 2011), action/intentions

338 understanding and imitation (the mirror neuron system; Cattaneo \& Rizzolatti, 2009;

339 Oberman \& Ramachandran, 2007) and the processing and integration of afferent sensory

340 information (thalamus; Halassa \& Kastner, 2017; Hwang, Bertolero, Liu, \& D'Esposito,

341 2017). All of these systems can be dysfunctional in ASD (Burrows, Laird, \& Uddin,

342 2016; Cheng, Rolls, Gu, Zhang, \& Feng, 2015; Dichter, Felder, \& Bodfish, 2009;

343 Hamilton, 2013; Linke, Keehn, Pueschel, Fishman, \& Müller, 2018; Padmanabhan,

344 Lynch, Schaer, \& Menon, 2017). The present study thus extends previous ones by

345 providing the first evidence that, at least for children included in the ABIDE datasets,

346 alterations in homotopic interhemispheric rsFC in large-scale brain networks have

347 already occurred in individuals aged from 5 to 10 years of age.

348 Furthermore, correlation analyses between the homotopic interhemispheric

349 functional connectivity and symptom severity revealed negative correlations between

350 interhemispheric functional connectivity of the MTG and total ADOS scores and between

351 homotopic interhemispheric connectivity of the MTG and the IOG and social sub-scale 
352 scores. This may indicate that reduced synchronization between the left and right parts of

353 the MTG and the IOG, as reflected by lower interhemispheric functional connectivity,

354 corresponds to greater symptom load in ASD. This is also consistent with negative

355 correlation patterns as found in our previous study involving primarily adults/adolescents

356 from the ABIDE-I dataset (Li et al., 2019) and may indicate a closer clinical relevance of

357 the homotopic interhemispheric connectivity of these regions specifically in ASD

358 children. In contrast, significant positive correlations were found between

359 interhemispheric functional connectivity of the PCC and total ADOS and social sub-scale

360 scores and between the precuneus and communication subscale scores. Similar positive

361 correlation patterns were also observed for the homotopic interhemispheric connectivity

362 of the PCC, the ITG, the precuneus and the IPL with the stereotyped behavior subscale

363 scores, indicating that stronger synchronization between the left and right parts of these

364 regions corresponds to greater symptom load in ASD. Thus, associations between

365 homotopic functional connectivity and symptom severity appear to be more complex in

366 ASD children, particularly the positive correlations with regions within the DMN,

367 contrast to some extent with our previous study using primarily adult/adolescent subjects

368 where we observed a negative association between homotopic interhemispheric PCC

369 functional connectivity and the ADOS communication sub-scale (Li et al., 2019). A study

370 on the developmental trajectories of homotopic functional connectivity strength in ASD

371 children has reported that some DMN regions show an increase in connectivity during the 
372 first decade of life, followed subsequently by a maintained decrease during adolescence

373 and adulthood (Kozhemiako et al., 2018). It is possible that initially a greater reduction in

374 the homotopic functional connectivity of the PCC and precuneus may offer some

375 compensatory benefit and reduced symptoms, but that subsequently in a more fully

376 developed brain in adolescence/adulthood there is benefit in increasing the homotopic

377 functional connectivity strength. This might explain the positive correlation with

378 symptom severity during childhood but negative correlation during

379 adolescence/adulthood and is in accord with positive correlations between homotopic

380 interhemispheric connectivity of the MTG and the IOG in the visual processing network

381 and ADOS scores as found in the present study and that homotopic functional

382 connectivity strength of visual processing regions in ASD show flatter developmental

383 trajectories relative to regions in DMN (Kozhemiako et al., 2018). Interestingly, the study

384 on homotopic developmental trajectories also reported a positive association with ADOS

385 scores as a function of developmental curvature coefficient (Kozhemiako et al., 2018).

386 However, some caution is required when interpreting this finding since ADOS scores

387 were only available for a relatively small number of ASD children in our current study.

388 Reduced homotopic functional connectivity has also been consistently reported in

389 another developmental psychiatric disorder, schizophrenia (Guo et al., 2013; Li, Xu,

390 Zhang, Hoptman, \& Zuo, 2015) suggesting that impaired interhemispheric connectivity

391 may be a hallmark of these disorders and contribute to some of the cognitive, social, 
392 motor and self-processing dysfunctions common to both. Decreased homotopic

393 functional connectivity has been observed in early onset schizophrenia patients (mean

394 age 14.5 years - Li et al., 2015) as well as adults (Guo et al., 2013), although specific

395 regions associated with symptom severity differ from those associated with symptom

396 severity in ASD.

397 We did not find significant differences between the ASD and TD children in CC

398 volume. Previous findings on CC volume changes in ASD patients have been

399 inconsistent, with some previous studies reporting reductions in both ASD adults and

400 children (Haar, Berman, Behrmann, \& Dinstein, 2016; Hardan et al., 2009; Keary et al.,

401 2019; Li et al., 2019) and others in contrast observing no significant alterations (Herbert

402 et al., 2014; Lefebvre, Beggiato, Bourgeron, \& Toro, 2015; Rice et al., 2005). The

403 inconsistency between these findings may well be as a result of many of the previous

404 studies being statistical underpowered (Lefebvre et al., 2015) and CC volume changes

405 derived from simple VBM-derived measures may also be too coarse an index to

406 characterize subtle changes in ASD (Lefebvre et al., 2015; Li et al., 2019).

407 There are some limitations in the present study. Firstly, the ages of the ASD and TD

408 children included in the present study ranged from 5 to 10 years old and therefore it is

409 possible that younger children might not show similar reduced homotopic functional

410 connectivity. This needs to be taken into consideration when making general inferences

411 based on findings concerning age-independency of homotopic connectivity changes. 
412 Secondly, the ABIDE dataset only includes high-functioning ASD patients, therefore the

413 current findings related to ASD children may not necessarily apply to low-functioning

414 ASD populations. Finally, ADOS measurements were only available for a relatively

415 small number of ASD children included in the study.

416 In conclusion, the present study using the ABIDE dataset has revealed decreased

417 homotopic interhemispheric functional connectivity in diffuse brain networks including

418 the DMN, the salience network, the mirror neuron system, the visual processing network,

419 and thalamus in the ASD relative to the TD children between the ages of 5 and 10 . Homo

420 topic functional connectivity changes between regions in the DMN and the visual

421 processing network were associated with the symptom severity. Thus, reduced homotopic

422 interhemispheric functional organization appears to be present in both high functioning

423 ASD children and adults/adolescents and may provide a useful general biomarker across

424 both early and late developmental stages of the disorder. 


\section{References}

427 Anderson, J. S., Druzgal, T. J., Froehlich, A., DuBray, M. B., Lange, N., Alexander, A.

428 L., ... Lainhart, J. E. (2011). Decreased interhemispheric functional connectivity in

429 autism. Cerebral Cortex, 21, 1134-1146. https://doi.org/10.1093/cercor/bhq190

430 Ashburner, J., \& Friston, K. J. (2000). Voxel-based morphometry-the methods.

$431 \quad$ Neuroimage, 11, 805-821. https://doi.org/10.1006/nimg.2000.0582

432 Bogousslavsky, J., Miklossy, J., Deruaz, J. P., Assal, G., \& Regli, F. (1987). Lingual and

433 fusiform gyri in visual processing: a clinico-pathologic study of superior altitudinal

434 hemianopia. Journal of Neurology, Neurosurgery \& Psychiatry, 50, 607-614.

435 http://dx.doi.org/10.1136/jnnp.50.5.607

436 Burrows, C. A., Laird, A. R., \& Uddin, L. Q. (2016). Functional connectivity of brain

437 regions for self- and other-evaluation in children, adolescents and adults with

438 autism. Developmental Science, 19, 564-580. https://doi.org/10.1111/desc.12400

439 Cattaneo, L., \& Rizzolatti, G. (2009). The mirror neuron system. Archives of Neurology,

$440 \quad 66,557-560$. https://doi.org/10.1146/annurev.neuro.27.070203.144230

441 Charman, T. (2003). Why is joint attention a pivotal skill in autism? Philosophical

442 transactions of the Royal Society of London. Series B, Biological sciences, 358, 315-

443 324. https://doi.org/10.1098/rstb.2002.1199

444 Chen, C. M., Yang, P., Wu, M. T., Chuang, T. C., \& Huang, T. Y. (2019). Deriving and

445 validating biomarkers associated with autism spectrum disorders from a large-scale 
resting-state database. Scientific Reports, 9, 9043. https://doi.org/10.1038/s41598-

448 Cheng, W., Rolls, E. T., Gu, H., Zhang, J. \& Feng, J. (2015). Autism: reduced connectivity between cortical areas involved in face expression, theory of mind and the sense of self. Brain, 138, 1382-1393. https://doi.org/10.1093/brain/awv051

451 Chevallier, C., Grèzes, J., Molesworth, C., Berthoz, S., \& Happé, F. (2012). Brief report:

455 Courchesne, E., \& Pierce, K. (2005). Why the frontal cortex in autism might be talking 0 only to itself: local over-connectivity but long-distance disconnection. Current Opinion in Neurobiology, 15, 225-230. https://doi.org/10.1016/j.conb.2005.03.001 \& Iacoboni, M. (2006). Understanding emotions in others: mirror neuron dysfunction in children with autism spectrum disorders. Nature Neuroscience, 9, 28mode network. Neuroimage, 132, 390-397.

465 Dichter, G. S., Felder, J. N., \& Bodfish, J. W. (2009). Autism is characterized by dorsal 
anterior cingulate hyperactivation during social target detection. Social Cognitive

468 Eickenberg, M., Gramfort, A., Varoquaux, G., \& Thirion, B. (2017). Seeing it all:

469 Convolutional network layers map the function of the human visual system.

471 Martino, A. D., Yan, C. G., Li, Q., Denio, E., Castellanos, F. X., Alaerts, K., ... evaluation of the intrinsic brain architecture in autism. Molecular Psychiatry, 19, 659-667. https://doi.org/10.1038/mp.2013.78

475 Ecker, C., Bookheimer, S. Y., \& Murphy, D. G. M. (2015). Neuroimaging in autism 476 spectrum disorder: brain structure and function across the lifespan. Lancet Neurology, 14, 1121-1134. https://doi.org/10.1016/S1474-4422(15)00050-2

478 Fedor, J., Lynn, A., Foran, W., DiCicco-Bloom, J., Luna, B., \& O'Hearn, K. (2018).

479 Patterns of fixation during face recognition: Differences in autism across age. Autism, 22, 866-880. https://doi.org/10.1177/1362361317714989

481 Fishman, I., Keown, C. L., Lincoln, A. J., Pineda, J. A., \& Müller, R. A. (2014). Atypical cross talk between mentalizing and mirror neuron networks in autism spectrum disorder. JAMA Psychiatry, 71, 751-760. doi:10.1001/jamapsychiatry.2014.83\%J JAMA Psychiatry

485 Frazier, T. W., \& Hardan, A. Y. (2009). A meta-analysis of the corpus callosum in 
autism. Biological Psychiatry, 66, 935-941.

488 Fortin, J. P., Cullen, N., Sheline, Y. I., Taylor, W. D., Aselcioglu, I., Cook, P. A., ... scanners and sites. Neuroimage, 167, 104-120.

Fortin, J. P., Parker, D., Tunç, B., Watanabe, T., Elliott, M. A., Ruparel, K., ... Schultz, R.

495 Friston, K. J., Williams, S., Howard, R., Frackowiak, R. S., \& Turner, R. (1996). resting-state functional magnetic resonance imaging. The Journal of Medical Investigation, 63, 204-208. https://doi.org/10.2152/jmi.63.204

502 Geschwind, D. H., \& Levitt, P. (2007). Autism spectrum disorders: developmental disconnection syndromes. Current Opinion in Neurobiology, 17, 103-111. https://doi.org/10.1016/j.conb.2007.01.009

505 Guo, S., Kendrick, K. M., Zhang, J., Broome, M., Yu, R., Liu, Z., \& Feng, J. (2013). 

schizophrenia and distinguishes it from depression. Neuroimage: Clinical, 2, 818826. https://doi.org/10.1016/j.nicl.2013.06.008

Haar, S., Berman, S., Behrmann, M., \& Dinstein, I. (2016). Anatomical abnormalities in autism. Cerebral Cortex, 26, 1440-1452. https://doi.org/10.1093/cercor/bhu242

511 Hagan, J. F. Jr, Shaw, J. S., \& Duncan, P., eds. (2008). Bright Futures: Guidelines for the

514 Halassa, M. M., \& Kastner, S. (2017). Thalamic functions in distributed cognitive control. Nature Neuroscience, 20, 1669-1679. https://doi.org/10.1038/s41593-0170020-1 systematic review of current theories. Developmental Cognitive Neuroscience, 3, 91-

520 Happé, F., \& Frith, U. (2006). The weak coherence account: detail-focused cognitive style in autism spectrum disorders. Journal of Autism and Developmental Disorders,

523 Hardan, A. Y., Pabalan, M., Gupta, N., Bansal, R., Melhem, N. M., Fedorov, S., ...

524 Minshew, N. J. (2009). Corpus callosum volume in children with autism. Psychiatry $525 \quad$ Research, 174, 57-61. https://doi.org/10.1016/j.rasd.2012.09.007 
526 Herbert, M. R., Ziegler, D. A., Makris, N., Filipek, P. A., Kemper, T. L., Normandin, J.

527 J., ... Caviness Jr, V. S. (2004). Localization of white matter volume increase in

528 autism and developmental language disorder. Annals of Neurology, 55, 530-540.

529 https://doi.org/10.1002/ana.20032

530 Herringshaw, A. J., Kumar, S. L., Rody, K. N., \& Kana, R. K. (2018). Neural Correlates

531 of Social Perception in Children with Autism: Local versus Global Preferences.

532 Neuroscience, 395, 49-59. https://doi.org/10.1016/j.neuroscience.2018.10.044

533 Hull, J. V., Jacokes, Z. J., Torgerson, C. M., Irimia, A., \& van Horn, J. D. (2017).

534 Resting-state functional connectivity in autism spectrum disorders: a review.

535 Frontiers in Psychiatry, 7, 205. https://doi.org/10.3389/fpsyt.2016.00205

536 Hwang, K., Bertolero, M. A., Liu, W. B., \& D'Esposito, M. (2017). The human thalamus

537 is an integrative hub for functional brain networks. Journal of Neuroscience, 37,

538 5594-5607. https://doi.org/10.1523/JNEUROSCI.0067-17.2017

539 Iacoboni, M., \& Dapretto, M. (2006). The mirror neuron system and the consequences of

$540 \quad$ its dysfunction. Nature Reviews Neuroscience, 7, 942-951.

$541 \quad$ https://doi.org/10.1038/nrn2024

542 Johnson, W. E., Li, C., \& Rabinovic, A. (2007). Adjusting batch effects in microarray

543 expression data using empirical Bayes methods. Biostatistics, 8, 118-127.

544 https://doi.org/10.1093/biostatistics/kxj037

545 Kanner, L. (1943). Autistic disturbances of affective contact. Nervous Child, 2, 217-250. 
546 Keary, C. J., Minshew, N. J., Bansal, R., Goradia, D., Fedorov, S., Keshavan, M. S., \&

547 Hardan, A. Y. (2009). Corpus callosum volume and neurocognition in autism.

$548 \quad$ Journal of Autism and Developmental Disorders, 39, 834-841.

549 https://doi.org/10.1007/s10803-009-0689-4

550 King, J. B., Prigge, M. B. D., King, C. K., Morgan, J., Weathersby, F., Chancellor Fox,

551 J., ... Anderson, J.S. (2019). Generalizability and reproducibility of functional

552 connectivity in autism. Molecular Autism,10, 27. https://doi.org/10.1186/s13229-

$553 \quad 019-0273-5$

554 Kozhemiako, N., Vakorin, V., Nunes, A. S., Iarocci, G., Ribary, U., \& Doesburg, S. M.

555 (2018). Extreme male developmental trajectories of homotopic brain connectivity.

556 Human Brain Mapping, 40, 987-1000. https://doi.org/10.1002/hbm.24427

557 Lancaster, J. L. (1997). The Talairach Daemon, a database server for Talairach atlas

$558 \quad$ labels. Neuroimage, 5, S633.

559 Lau, W., Leung, M. K., \& Lau, B. (2019). Resting-state abnormalities in Autism

$560 \quad$ Spectrum Disorders: A meta-analysis. Scientific Reports, 9, 3892.

$561 \quad$ https://doi.org/10.1038/s41598-019-40427-7

562 Lee, Y., Park, B. Y., James, O., Kim, S. G., \& Park, H. (2017). Autism Spectrum

563 Disorder Related Functional Connectivity Changes in the Language Network in

564 Children, Adolescents and Adults. Frontiers in Human Neuroscience, 11, 418.

565 https://doi.org/10.3389/fnhum.2017.00418 
566 Lefebvre, A., Beggiato, A., Bourgeron, T., \& Toro, R. (2015). Neuroanatomical diversity

567 of corpus callosum and brain volume in autism: meta-analysis, analysis of the

568 autism brain imaging data exchange project, and simulation. Biological Psychiatry,

$569 \quad 78,126-134$. https://doi.org/10.1016/j.biopsych.2015.02.010

570 Li, H. J., Xu, Y., Zhang, K. R., Hoptman, M. J., \& Zuo, X. N. (2015). Homotopic

571 connectivity in drug-naïve, first-episode, early-onset schizophrenia. Journal of

572 Child Psychology and Psychiatry, 56, 432-443. https://doi.org/10.1111/jcpp.12307

573 Li, Q., Becker, B., Jiang, X., Zhao, Z., Zhang, Q., Yao, S., \& Kendrick, K. M. (2019).

574 Decreased interhemispheric functional connectivity rather than corpus callosum

575 volume as a potential biomarker for autism spectrum disorder. Cortex, 119, 258-266.

$576 \quad$ https://doi.org/10.1016/j.cortex.2019.05.003

577 Linke, A. C., Keehn, R. J. J., Pueschel, E. B., Fishman, I., \& Müller, R. A. (2018).

$578 \quad$ Children with ASD show links between aberrant sound processing, social

579 symptoms, and atypical auditory interhemispheric and thalamocortical functional

580 connectivity. Developmental Cognitive Neuroscience, 29, 117-126.

$581 \quad$ https://doi.org/10.1016/j.den.2017.01.007

582 Machielsen, W. C., Rombouts, S. A., Barkhof, F., Scheltens, P., \& Witter, M. P. (2000).

583 FMRI of visual encoding: reproducibility of activation. Human Brain Mapping, 9,

584 156-164. https://doi.org/10.1002/(SICI)1097-0193(200003)9:3<156::AID-

$585 \quad$ HBM4>3.0.CO;2-Q 
586 MacDonald, A. W., Cohen, J. D., Stenger, V. A., \& Carter, C. S. (2000). Dissociating the

587 role of the dorsolateral prefrontal and anterior cingulate cortex in cognitive control.

$588 \quad$ Science, 288, 1835-1838. https://doi.org/10.1126/science.288.5472.1835

589 Madipakkam, A. R., Rothkirch, M., Dziobek, I., \& Sterzer, P. (2017). Unconscious

590 avoidance of eye contact in autism spectrum disorder. Scientific Reports, 7, 13378.

$591 \quad$ https://doi.org/10.1038/s41598-017-13945-5

592 Menon, V., \& Uddin, L. Q. (2010). Saliency, switching, attention and control: a network

593 model of insula function. Brain Structure and Function, 214, 655-667.

594 https://doi.org/10.1007/s00429-010-0262-0

595 Monk, C. S., Peltier, S. J., Wiggins, J. L., Weng, S. J., Carrasco, M., Risi, S., \& Lord, C.

596 (2009). Abnormalities of intrinsic functional connectivity in autism spectrum

597 disorders. Neuroimage, 47, 764-772.

598 https://doi.org/10.1016/j.neuroimage.2009.04.069

599 Morrison, K. E., Pinkham, A. E., Penn, D. L., Kelsven, S., Ludwig, K., \& Sasson, N. J.

600 (2017). Distinct profiles of social skill in adults with autism spectrum disorder and

601 schizophrenia. Autism Research, 10, 878-887. https://doi.org/10.1002/aur.1734

602 Oberman, L. M., \& Ramachandran, V. S. (2007). The simulating social mind: the role of

603 the mirror neuron system and simulation in the social and communicative deficits of

604 autism spectrum disorders. Psychological Bulletin, 133, 310.

605 https://doi.org/10.1037/0033-2909.133.2.310 
606 Oberwelland, E., Schilbach, L., Barisic, I., Krall, S. C., Vogeley, K., Fink, G. R., ...

607 Schulte-Rüther, M. (2017). Young adolescents with autism show abnormal joint

608 attention network: A gaze contingent fMRI study. Neuroimage: Clinical, 14, 112-

609 121. https://doi.org/10.1016/j.nicl.2017.01.006

610 Padmanabhan, A., Lynch, C. J., Schaer, M., \& Menon, V. (2017). The default mode

611 network in autism. Biological Psychiatry, 2, 476-486.

$612 \quad$ https://doi.org/10.1016/j.bpsc.2017.04.004

613 Power, J. D., Mitra, A., Laumann, T. O., Snyder, A. Z., Schlaggar, B. L., \& Petersen, S.

614 E. (2014). Methods to detect, characterize, and remove motion artifact in resting

615 state fMRI. Neuroimage, 84, 320-341.

616 https://doi.org/10.1016/j.neuroimage.2013.08.048

617 Raichle, M. E. (2015). The brain's default mode network. Annual Review of

618 Neuroscience, 38, 433-447. https://doi.org/10.1146/annurev-neuro-071013-014030

619 Raichle, M. E., MacLeod, A. M., Snyder, A. Z., Powers, W. J., Gusnard, D. A., \&

620 Shulman, G. L. (2001). A default mode of brain function. Proceedings of the

$621 \quad$ National Academy of Sciences, 98, 676-682. doi:10.1073/pnas.98.2.676

622 Rice, S. A., Bigler, E. D., Cleavinger, H. B., Tate, D.F., Sayer, J., McMahon, W.,

623 Ozonoff, S., Lu, J., \& Lainhart, J. E. (2005). Macrocephaly, corpus callosum

624 morphology, and autism. Journal of Child Neurology, 20, 34-41.

625 https://doi.org/10.1177/08830738050200010601 
626 Rizzolatti, G., \& Craighero, L. (2004). The mirror-neuron system. Annual Review of

$627 \quad$ Neuroscience, 27, 169-192.

628 https://doi.org/10.1146/annurev.neuro.27.070203.144230

629 Satterthwaite, T. D., Elliott, M. A., Gerraty, R. T., Ruparel, K., Loughead, J., Calkins, M.

630 E., ... Wolf, D.H. (2013). An improved framework for confound regression and

631 filtering for control of motion artifact in the preprocessing of resting-state functional

632 connectivity data. Neuroimage, 64, 240-256.

633 https://doi.org/10.1016/j.neuroimage.2012.08.052

634 Seghier, M. L. (2013). The angular gyrus: multiple functions and multiple subdivisions.

635 The Neuroscientist, 19, 43-61. https://doi.org/10.1177/1073858412440596

636 Stark, D. E., Marguiles, D. S., Shehzad, Z. E, Reiss, P., Clare Kelly, A. M. C., ...

637 Milham, M.P. (2008). Regional variation in interhemispheric coordination of

638 intrinsic hemodynamic fluctuations. Journal of Neuroscience, 28, 13754-13764.

639 https://doi.org/10.1523/JNEUROSCI.4544-08.2008

640 Stevens, F. L., Hurley, R. A., \& Taber, K. H. (2011). Anterior cingulate cortex: unique

641 role in cognition and emotion. The Journal of Neuropsychiatry and Clinical

$642 \quad$ Neurosciences, 23, 121-125. doi:10.1001/jamapsychiatry.2013.104

643 Supekar, K., Kochalka, J., Schaer, M., Wakeman, H., Qin, S., Padmanabhan, A., \&

644 Menon, V. (2018). Deficits in mesolimbic reward pathway underlie social

645 interaction impairments in children with autism. Brain, 141, 2795-2805. 
647 Uddin, L. Q., Supekar, K., Lynch, C. J., Khouzam, A., Phillips, J., Feinstein, C., ...

648 Menon, V. (2013). Salience network-based classification and prediction of symptom

649 severity in children with autism. JAMA Psychiatry, 70, 869-879.

doi:10.1001/jamapsychiatry.2013.104

651 Vidal, C. N., Nicolson, R., DeVito, T. J., Hayashi, K. M., Geaga, J. A., Drost, D. J., ...

652 Thompson, P.M. (2006). Mapping corpus callosum deficits in autism: an index of

653 aberrant cortical connectivity. Biological Psychiatry, 60, 218-225.

654 https://doi.org/10.1016/j.biopsych.2005.11.011

655 von dem Hagen, E. A., Stoyanova, R. S., Rowe, J. B., Baron-Cohen, S., \& Calder, A. J.

656 (2014). Direct gaze elicits atypical activation of the theory-of-mind network in

657 autism spectrum conditions. Cerebral Cortex, 24, 1485-1492.

658 https://doi.org/10.1093/cercor/bht003

659 Wang, A. T., Dapretto, M., Hariri, A. R., Sigman, M., \& Bookheimer, S. Y. (2004).

660 Neural correlates of facial affect processing in children and adolescents with autism

661 spectrum disorder. Journal of the American Academy of Child and Adolescent

$662 \quad$ Psychiatry, 43, 481-490. https://doi.org/10.1097/00004583-200404000-00015

663 Washington, S. D., Gordon, E. M., Brar, J., Warburton, S., Sawyer, A. T., Wolfe, A., ...

664 VanMeter, J.W. (2014). Dysmaturation of the default mode network in autism.

665 Human Brain Mapping, 35, 1284-1296. https://doi.org/10.1002/hbm.22252 
666 Wegiel, J., Kaczmarski, W., Flory, M., Martinez-Cerdeno, V., Wisniewski, T., Nowicki,

667 K., Kuchna, I., \& Wegiel, J. (2018). Deficit of corpus callosum axons, reduced axon

668 diameter and decreased area are markers of abnormal development of

669 interhemispheric connections in autistic subjects. Acta Neuropathologica

670 Communications, 6, 143. https://doi.org/10.1186/s40478-018-0645-7

671 Wilke, M., Holland, S. K., Altaye, M., \& Gaser, C. (2008). Template-O-Matic: a toolbox

672 for creating customized pediatric templates. Neuroimage, 41, 903-913.

673 https://doi.org/10.1016/j.neuroimage.2008.02.056

674 Yan, C., \& Zang, Y. (2010). DPARSF: a MATLAB toolbox for "pipeline" data analysis

675 of resting-state fMRI. Frontiers in Systems Neuroscience, 4, 13.

676 https://doi.org/10.3389/fnsys.2010.00013

677 Yan, C. G., Craddock, R. C., Zuo, X. N., Zang, Y. F., \& Milham, M. P. (2013).

678 Standardizing the intrinsic brain: towards robust measurement of inter-individual

679 variation in 1000 functional connectomes. Neuroimage, 80, 246-262.

680 https://doi.org/10.1016/j.neuroimage.2013.04.081

681 Yu, M., Linn, K. A., Cook, P. A., Phillips, M. L., McInnis, M., Fava, M., ... Sheline, Y. I.

682 (2018). Statistical harmonization corrects site effects in functional connectivity

683 measurements from multi-site fMRI data. Human Brain Mapping, 39, 4213-4227.

684 https://doi.org/10.1002/hbm.24241 
685 Zuo, X. N., Kelly, C., Di Martino, A., Mennes, M., Margulies, D. S., Bangaru, S., ...

686 Milham, M. P. (2010). Growing together and growing apart: regional and sex

687 differences in the lifespan developmental trajectories of functional homotopy.

$688 \quad$ Journal of Neuroscience, 30, 15034-15043.

689 https://doi.org/10.1523/JNEUROSCI.2612-10.2010

690 
Table 1. Regions showing decreased interhemispheric connectivity in the autism relative to the control groups (MNI coordinates).

\begin{tabular}{|c|c|c|c|c|c|c|}
\hline Brain Region & BA & No. Voxels & Peak t-value & $\mathrm{x}$ & $\mathrm{y}$ & $\mathrm{z}$ \\
\hline Posterior Cingulate Cortex & $31 / 7 / 23$ & 753 & 4.88 & 6 & -45 & 12 \\
\hline Posterior Cingulate Cortex & & & 4.77 & 6 & -42 & 24 \\
\hline Precuneus & & & 3.91 & 6 & -60 & 27 \\
\hline Lingual Gyrus & & & 3.84 & 27 & -54 & -6 \\
\hline Midbrain & & & 3.47 & 3 & -27 & -6 \\
\hline Fusiform & & & 3.16 & 30 & -69 & -3 \\
\hline Thalamus & & & 2.93 & 12 & -30 & 3 \\
\hline Middle Temporal Gyrus & 21 & 69 & 4.31 & 66 & -18 & -9 \\
\hline Middle Occipital Gyrus & $18 / 19$ & 106 & 4.28 & 30 & -99 & 3 \\
\hline Inferior Occipital Gyrus & & & 3.10 & 33 & -90 & -6 \\
\hline Anterior Cingulate Cortex & $24 / 32$ & 41 & 4.05 & 6 & 36 & 3 \\
\hline Ventral Medial Prefrontal Cortex & & & 2.27 & 6 & 36 & -9 \\
\hline Inferior Temporal Gyrus & $37 / 20$ & 32 & 4.02 & 66 & -54 & -12 \\
\hline Middle Temporal Gyrus & & & 3.45 & 54 & -48 & -12 \\
\hline Caudate & & 63 & 3.61 & 15 & 6 & 18 \\
\hline Dorsal Medial Prefrontal Cortex & 10 & 42 & 3.50 & 9 & 63 & 9 \\
\hline Supramarginal/Angular Gyrus & 40 & 32 & 3.49 & 60 & -51 & 33 \\
\hline Precentral Gyrus & 6 & 23 & 3.46 & 36 & -15 & 42 \\
\hline Middle Frontal Gyrus & $8 / 6$ & 84 & 3.46 & 27 & 18 & 51 \\
\hline Postcentral Gyrus & 5 & 44 & 3.22 & 24 & -42 & 63 \\
\hline Precuneus & 7 & 58 & 3.18 & 21 & -60 & 51 \\
\hline Inferior Parietal Lobule & & & 2.50 & 36 & -60 & 48 \\
\hline
\end{tabular}

691 All with a $\mathrm{p}<0.05$ FDR corrected threshold and voxels larger than 10 corrected within the mask from Li et al.

692 (2019). BA: Brodmann Area. Brain coordinates are reported in the Montreal Neurological Institute (MNI) space. 


\section{Figure Legends}

695 Figure 1. Regions showing significantly $\left(\mathrm{p}_{\mathrm{FDR}}<0.05\right)$ decreased homotopic interhemispheric

696 functional connectivity in the ASD compared to the typically developing groups. PCC $=$

697 posterior cingulate cortex; $\mathrm{dmPFC}=$ dorsomedial prefrontal cortex $; \mathrm{vmPFC}=$ ventromedial

698 prefrontal cortex; PreCG = precentral gyrus; $I O G$ = inferior occipital gyrus; LING = lingual

699 gyrus; $\mathrm{FFG}=$ fusiform gyrus; PostCG = postcentral gyrus; IPL = inferior parietal lobule. Color

700 bar indicates $t$ values of the statistical map.

701 Figure 2. Correlations between the homotopic interhemispheric functional connectivity strengths of

702 the posterior cingulate cortex (PCC) (A) and the middle temporal gyrus (MTG) (B) and ADOS total

703 scores. Correlations between the homotopic connectivity strengths of the PCC and social sub-scale

704 scores (C), and of the precuneus (D) for the communication sub-scale scores. A consistent positive

705 correlation pattern was found between the homotopic connectivity of the PCC (E), the inferior

706 temporal gyrus $(\mathrm{F})$, the precuneus $(\mathrm{G})$ and the inferior parietal lobule $(\mathrm{H})$ and stereotyped behavior

707 sub-scale scores. However, the homotopic connectivity strengths of the MTG (I) and inferior occipital

708 gyrus (J) were negatively associated with social sub-scale scores. 

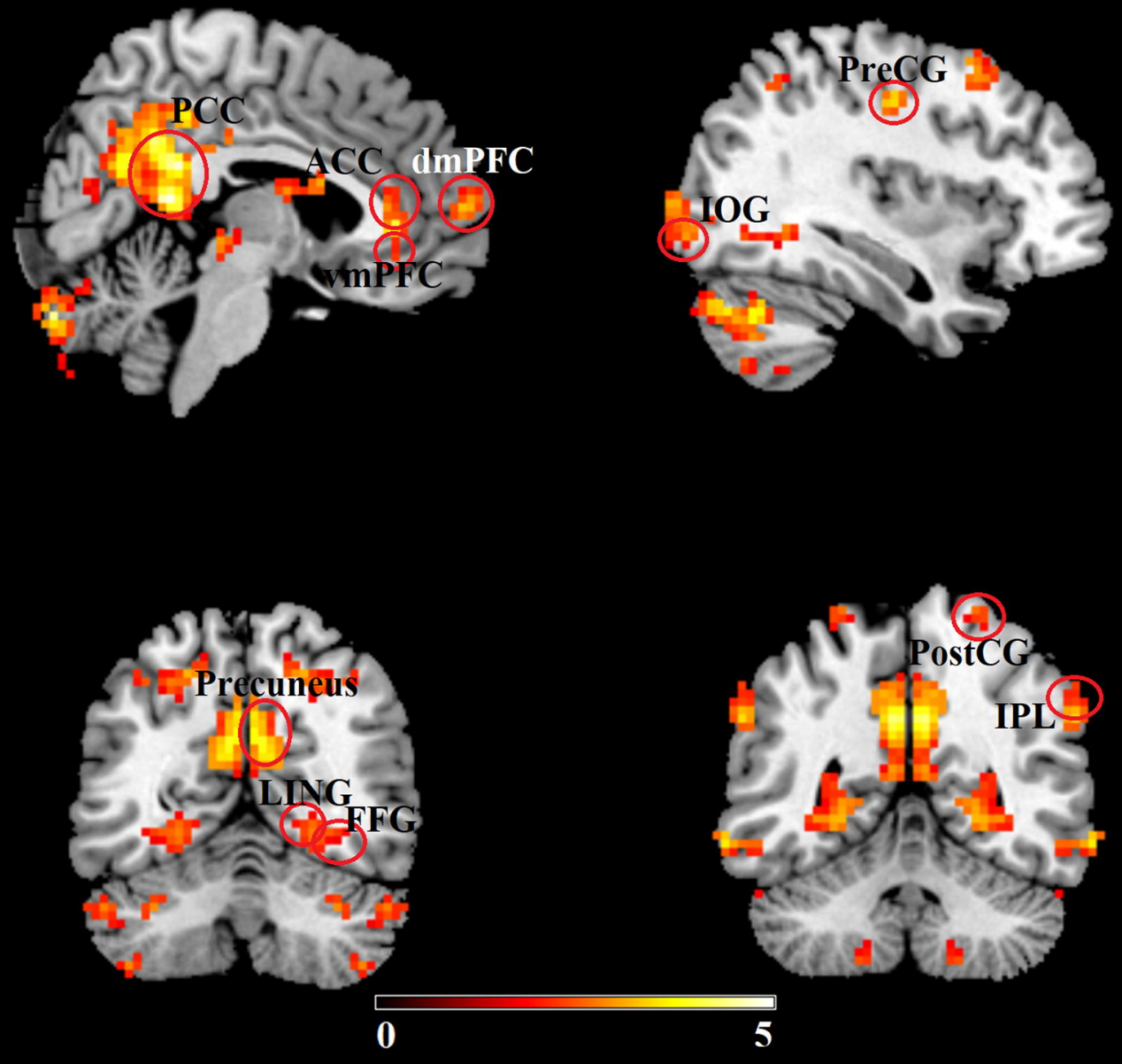
